\title{
Sacubitril/valsartan Decreased Atrial Fibrillation Susceptibility by Inhibiting Angiotensin II-induced Atrial Fibrosis through p-Smad2/3, p-JNK, and p-p38 Signaling Pathways
}

\section{Song-Nan Li}

Capital Medical University Affiliated Anzhen Hospital

Jing-Rui Zhang

Department of Cardiology, Beijing Anzhen Hospital, Capital Medical University, Beijing, China Hui Xi

Department of Cardiology, Peking University International Hospital, Beijing, China

Chang-Yi Li (D lichangyi1212@sina.com )

Department of Cardiology, Beijing Anzhen Hospital, Capital Medical University, Beijing, China Lei Zhao

Department of Radiololgy, Beijing Anzhen Hospital, Capital Medical University, Beijing, China

\section{Research}

Keywords: sacubitril/valsartan, angiotensin II, atrial fibrosis, atrial fibrillation

Posted Date: November 19th, 2020

DOI: https://doi.org/10.21203/rs.3.rs-110578/v1

License: (a) (1) This work is licensed under a Creative Commons Attribution 4.0 International License.

Read Full License 


\section{Abstract}

Background: Sacubitril/valsartan (SAC/VAL), combined inhibitors of angiotensin receptor (AT-R) and neprilysin receptor, prevents Angiotensin $\otimes(A n g \otimes)$ from binding $A T_{1}-R$ and blocks degradation of natriuretic peptides. Despite its efficacy in reducing ventricular fibrosis and preserving cardiac functions, which has been extensively demonstrated in myocardial infarction or pressure overload models, few studies have been conducted to determine whether SAC/VAL could attenuate atrial fibrosis and decrease atrial fibrillation (AF) susceptibility.

Methods: Sprague-Dawley rats were divided into three groups after implantation of an osmotic pump preloaded with Ang $\triangle$ and received corn oil, VAL, or SAC/VAL treatment for 28 days. Electrophysiological study was performed to determine inducibility and duration of AF. Echocardiography was performed before and 28 days after osmotic mini-pump implantation to evaluate cardiac functions. Enzyme-linked immunosorbent assay was perfomed to detect the serum concentration of atrial natriuretic peptide (ANP), $\mathrm{N}$-terminal pro-brain natriuretic peptide (NT-proBNP) and Ang $\llbracket$. Masson staining was performed to determine the extent of interstitial fibrosis. Immunohistochemical, and immunofluorescence staining as well as transwell and MTT assay were also performed. Western blot analysis was perfomed to figure out how SAC/VAL exerts its anti-fibrosis effects in atriums.

Results: After 28 days of Ang $\llbracket$ stimulation, rats in SAC/VAL group exhibited reduced reduced extent of atrial fibrosis, inhibited proliferation, migration, and differentiation of atrial fibroblasts and decreased

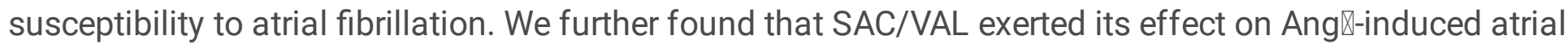
fibrosis by inhibiting the $\mathrm{p}-\mathrm{Smad} 2 / 3, \mathrm{p}-\mathrm{JNK}$, and p-p38 MAPK signaling pathways in vivo.

Conclusions: Our study provided experimental evidence for the inhibition of atrial fibrosis and reduced susceptibility to AF by SAC/VAL. These results emphasize the importance of SAC/VAL in the prevention of Ang囚-induced atrial fibrosis and may help to enrich the options for atrial fibrillation pharmacotherapy.

\section{Background}

As one of the most frequently encountered rhythm disturbances in the clinical setting, atrial fibrillation (AF) aggrandizes stroke risk and worsens the prognosis[1, 2]. Currently, available treatment options for AF fail to achieve satisfactory therapeutic benefits as the complex mechanisms behind this disease, including structural and electrical remodeling and autonomic nervous system dysfunction[3-6]. Notable among these mechanisms is atrial structural remodeling, which is perhaps a vital link of all the AF mechanisms. The development of atrial structural remodeling is strongly associated with atrial fibrosis[7]. Studies in this regard have identified that therapies that target atrial fibrosis would be of great clinical importance in treating AF[8-10].

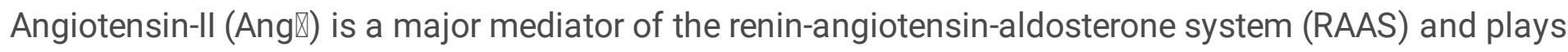
an essential role in the formation of atrial fibrosis[11, 12]. Upon binding to its type 1 receptor $\left(A T_{1}-R\right)$,

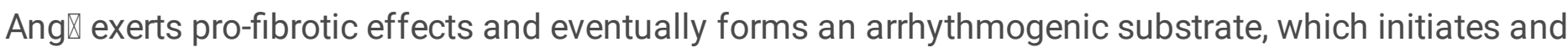


perpetuates AF[13]. The higher the Ang $\mathbb{\|}$ level, the more severe the extent of atrial fibrosis and the increased incidence of atrial fibrillation[14]. Growing evidence from animal studies has affirmed that blocking RAAS using angiotensin-converting enzyme inhibitors (ACEIs) and angiotensin receptor blockers (ARBs) reverse atrial fibrosis and decrease atrial arrhythmia inducibility[15, 16].

Sacubitril/valsartan (SAC/VAL), the first representative of a first-in-class drug, combined inhibitors of angiotensin receptor and neprilysin (neutral endopeptidase, NEP) receptor, prevents Ang $₫$ from binding to $A T_{1}-R$ and blocks degradation of natriuretic peptides (NPs)[17]. The PARADIGM-HF trial has evaluated its efficacy in reducing mortality and hospitalization among patients with heart failure with reduced ejection fraction (HFrEF)[18]. Previous experimental studies have demonstrated that SAC/VAL deterred the ventricular structural remodeling and dysfunction post-myocardial infarction (MI). Recently, Chang et al. used a rabbit MI and HF model to demonstrate SAC/VAL could preserve heart systolic function and avert Ml-induced electrophysiologic remodeling by reducing phosphorylated expression calmodulin-dependent protein kinase II (p-CaMKII)[19]. Vaskova et al. showed that downregulation of miR-181a exosomes could also be one of the mechanisms by which SAC/VAL improves cardiac function and reduces myocardial fibrosis in rats with chronic MI[20]. Protein kinase G (PKG) signaling and nuclear factor kappa-light-chainenhancer of activated B cells (NF-KB)/nucleotide-binding oligomerization domain (NOD)-like receptor protein 3 (NLRP3) signaling pathways are also involved in the attenuation of ventricular fibrosis by SAC/VAL[21, 22]. However, all of these published data on the signaling pathways through which SAC/VAL exerts its antifibrotic effects are heterogeneous. Moreover, it is still unclear that the particular mechanisms behind these protective roles of SAC/VAL on AF. Therefore, in this study, we aim to use a rat model with atrial fibrosis induced by Ang $₫$ continuous subcutaneous infusion to determine the effects of SAC/VAL on atrial fibrosis and elucidate the underlying mechanisms.

\section{Methods}

\subsection{Experimental Animals}

Adult male Sprague-Dawley rats (body weight 200-250 g) were purchased from SiPeiFu (Beijing) Biotechnology Co., Ltd. (Beijing, China). All animal studies were conducted in compliance with the Animal Care and Use Committee of Capital Medical University and the Guide for the Care and Use of Laboratory Animals published by the National Institutes of Health (the 8th Edition, NRC 2011).

\subsection{Osmotic mini-pump implantation, drug administration, and blood pressure measurement}

All SD rats were anesthetized with isoflurane (5\% induction; $2-3 \%$ maintenance). An osmotic pump

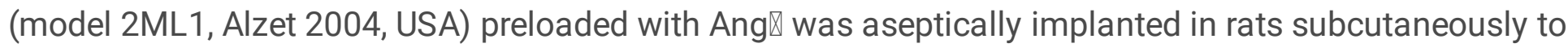
induce atrial fibrosis by the continuous release of Ang $\rrbracket(750 \mathrm{ng} / \mathrm{kg} / \mathrm{min})$ for 28 days. Later, all rats were assigned to three groups to receive corn oil, valsartan (VAL, $48 \mathrm{mg} / \mathrm{kg}$ body weight, $\mathrm{n}=6$ ), or sacubitril/valsartan (SAC/VAL, $60 \mathrm{mg} / \mathrm{kg}$ body weight, $n=6$ ) for 28 days. All drugs were dissolved in corn oil and administrated by gavage. Systolic blood pressure (SBP), diastolic blood pressure (SBP), and mean 
blood pressure (MBP) were measured at the end of experiment by the tail-cuff method as previously described[23].

\subsection{Electrophysiological study}

The electrophysiological study (EPS) was performed as previously described before the rats were sacrificed[24]. Briefly, rats were anesthetized with isoflurane (5\% induction; $2-3 \%$ maintenance) and placed on the heated pad to maintain body temperature at $37^{\circ} \mathrm{C}$. An electrode catheter $(1.1 \mathrm{~F}$, Science) was introduced into the right atrium via the right jugular vein then released a train of electrical stimuli at a pacing cycle length of $100 \mathrm{~ms}$ to test the susceptibility to atrial arrhythmias. Electrical stimulation was performed ten times in the same manner, and the number of successful AF induction within these ten times was recorded. The duration of $A F$ is determined by the time from the end of the burst pacing to the first sinus $P$ wave after the atrial rhythm. AF in this study was defined as a rapid and irregular atrial rhythm with an irregular R-R interval lasting more than $1000 \mathrm{~ms}$.

\subsection{Echocardiography}

Before and 28 days after osmotic mini-pump containing Ang囚 implantation, rats were examined with $\mathrm{M}$ mode echocardiography (Vevo 770, Visual Sonics, Inc., Toronto, Ontario, Canada). Inhalational anesthesia with $2 \%$ isoflurane and a heated pad were adopted during the process of image acquisition. Left atrial diameter (LAD), LV end-diastolic diameter (LVEDD), and ejection fraction (EF) were measured and averaged over three cardiac cycles. Mitral valve flow was assessed by using pulsed-wave Doppler ultrasonography. The early (E) and atrial (A) peaks are measured and averaged over three cardiac cycles.

\subsection{Enzyme-linked immunosorbent assay}

The serum concentration of atrial natriuretic peptide (ANP), N-terminal pro-brain natriuretic peptide (NTproBNP) and Ang $\triangle$ were detected by ELISA according to the manufacturer's instructions. Kits with Catalog E02A0204, E02A0493, and E02N0008 were purchased from Shanghai BlueGene Biotech Co., Ltd.. The optical density (OD) at $450 \mathrm{~nm}$ was measured by an ELISA reader. The results were expressed as $\mathrm{pg} / \mathrm{ml}$ or $\mathrm{ng} / \mathrm{ml}$.

\subsection{Histological, immunohistochemical, and immunofluorescence staining}

Left atrial tissue samples from rats were fixed with $4 \%$ paraformaldehyde, embedded in paraffin, transversely cut into $5 \mu \mathrm{m}$ thickness. Masson staining was performed as previously described to highlight the fibers, and the extent of interstitial fibrosis was determined by fibrosis area / total administration area $\times 100 \%$. Immunohistochemical staining were performed using antibodies against collagen I (Abcam, ab34710) and collagen III (Abcam, ab7778) as previously described. Immunofluorescence staining were performed using the methods described previously with antibodies against a-smooth muscle actin (aSMA, Abcam, ab5694) and vimentin (Abcam, ab137321). Images acquisition and analysis were used by Image-Pro Plus.

\subsection{Cell migration and proliferation evaluation}

Rat atrial fibroblasts (AFBs, Cat NO.: CP-R074) were purchased from Procell Life Science\&Technology Co.,Ltd (Wuhan, China) for cell proliferation and migration evaluation. The AFBs were divided into three 
groups and pretreated with dimethyl sulfoxide (MedChem Express, control group), valsartan (10 $\mu \mathrm{mol} / \mathrm{L}$, MedChem Express, VAL group), and LBQ657 + valsartan ( $10 \mu \mathrm{mol} / \mathrm{L}, 1: 1$ ratio, MedChem Express,

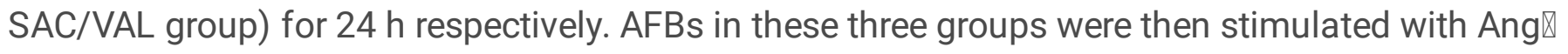
$(100 \mathrm{nmol} / \mathrm{L})$ for another $24 \mathrm{~h}$. Transwell assay was performed to evaluate cell migration. The nonmigratory cells remaining in the upper chamber were removed, and the membranes containing AFBs were fixed and then stained with crystal violet. Five fields at $\times 200$ magnification were randomly selected under the light microscope to count the cells migrating to the lower compartment. Cell proliferation was quantified using the 3-(4,5-dimethylthiazol-2-yl)-2,5-diphenyltetrazolium Bromide (MTT) assay (Sigma, St. Louis, MO, USA). The OD value at a wavelength of $490 \mathrm{~nm}$ of each well was detected using a spectrophotometer (BioTek, Elx800, Winooski, VT, USA).

\subsection{Western Blotting Analysis}

RIPA lysis buffer containing protease and phosphatase inhibitors were added to the proteins extracted from the left atrium of rats and quantified using the BCA protein analysis kit, as described previously. The following primary antibodies against phospho-Smad1/5/9 (CST, 13820), phospho-Smad2/3 (CST, 8828), p38 MAPK (CST, 8690S), phospho-p38 MAPK (CST, 9216S), ERK1/2 (CST, 4695S), phospho-ERK1/2 (CST, 9101S), JNK (CST, 9252S), phospho-JNK (CST, 4668S), and GAPDH (CST, 5174) were used for western blotting. Gel Imaging System (Tanon 5200) was used to detect the bands and the results were quantified by densitometry software (Image-Pro Plus).

\subsection{Statistical Analysis}

All data were represented as $\bar{x} \pm S D$ or percentage and analyzed using GraphPad Prism (version 6.0). One-way ANOVA followed by Tukey's post-hoc was used to determine statistical significance for multiple groups. A $P$ value $<0.05$ was considered statistically significant.

\section{Results}

\subsection{Sacubitril/valsartan attenuated Ang冈-induced atrial fibrosis}

To determine the effects of SAC/VAL on atrial fibrosis, the osmotic pump containing Ang $₫$ was implanted subcutaneously into rats to generate the atrial fibrosis model. Pathological assessment using Masson's staining and immunohistochemistry of collagen I and collagen III deposition were evaluated at day 28 after the procedure. In the saline group, Masson's staining revealed significant LA fibrosis in response to

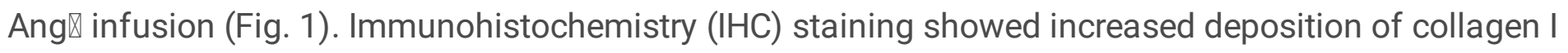
and collagen III (Fig. 1). While both SAC/VAL or VAL alone significantly restored histological changes and decreased collagen deposition in the atriums of Ang $₫$ stimulated rats. The quantitative morphometric analysis demonstrated that the extent of fibrosis and collagen I and collagen III depositions were significantly reduced in the rats from both VAL and SAC/VAL group compared to saline-treated rats $(\mathrm{P}<$ 0.05). Further analysis showed that the fibrotic extent and collagen deposition were significantly lower with SAC/VAL treatment than with VAL, suggesting that SAC/VAL possesses more potent antifibrotic capacity over VAL. 


\subsection{Sacubitril/valsartan inhibit the proliferation, migration, and differentiation of atrial fibroblasts}

We next used the MTT assay and the Transwell test to verify the effect of SAC/VAL on the migration and proliferation of atrial fibroblasts. Ang $₫$ promoted more remarkable cell migration and proliferation in the control group. As illustrated in Figs. 2B and 2C, the migration and proliferative rate of AFBs was significantly reduced after SAC/VAL or VAL alone administration. Compared with the VAL group, SAC/VAL had a stronger inhibitory effect on AFBs migration and proliferation. These results indicated that SAC/VAL might play roles in the function of AFBs in vitro.

To further confirm the protective effects of SAC/VAL, the immunofluorescence assay was carried out (Fig. 3). Results clearly revealed the increased expression of a-SMA and vimentin in atriums from salinetreated rats after Ang $\triangle$ stimulation. While in SAC/VAL and VAL treated rats, the distribution and expression of both a-SMA and vimentin were decreased (Fig. 3). Furthermore, a more obvious attenuation was observed in the SAC/VAL group. This result was consistent with the findings obtained by Masson's staining and IHC staining.

\subsection{Sacubitril/valsartan preserved atrial and ventricular functions}

We performed echocardiography to assess the systolic and diastolic function of the heart, as well as the left atrial dimension on the day before the procedure and the 28-day after the procedure. No difference was found in baseline parameters between all three groups (Fig. 4C-4F). After 28 days of Ang $\mathbb{\text { infusion, }}$ rats in the saline group developed marked LA enlargement, but not in SAC/VAL or VAL treated rats. The LVEDD and LVEF were increased, and the MV E/A ratio was decreased in saline-treated rats post-Ang $\mathbb{Z}$ stimulation. Such changes were corrected in the SAC/VAL, or VAL alone treated rats $(p<0.05)$ (Fig. 4). No difference was found in the abovementioned parameters between the two treated regimen. These findings were corroborated that previous clinical trials had demonstrated that SAC/VAL could improve

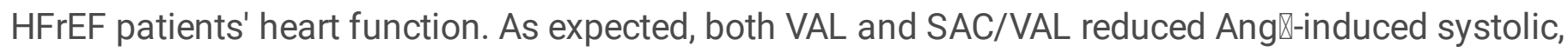
diastolic, and mean blood pressure increases compared with saline, but no difference in the two drugs' anti-hypertensive effects was observed. The serum concentrations of ANP, NT-proBNP, and Ang $\rrbracket$ were measured at the end of the experimental period. The level of ANP was elevated significantly in SAC/VAL group as expected (Fig. 5A). In addition, SAC/VAL therapy resulted in a significant reduction of serum NTproBNP compared to the control and VAL group (Fig. 6C). This result was consistent with changes in left ventricular systolic function. We also observed a tendency for an increase in the level of Ang $\triangle$ of SAC/VAL treatment, but there was no statistical significance (Fig. 6B).

\subsection{Sacubitril/valsartan decreased atrial arrhythmias inducibility}

We next tested whether marked atrial fibrosis post-Ang $₫$ stimulation could lead to a lower threshold for inducibility of AF. We performed in vivo electrophysiologic study (EPS), as mentioned previously[24]. In the whole process of EPS, all of the rats did not show a spontaneous arrhythmia. Saline treated rats showed significantly increased vulnerability to AF, as evidenced by an increased number of AF episodes and lengthier AF duration compared to VAL alone and SAC/VAL treated rats after two rounds of $30 \mathrm{~s}$ burst pacing (Fig. $5, p<0.05$ ). While the SAC/VAL group were more resistant to the AF-provoking effect of 
Ang $₫$ than VAL alone treated rats $(p<0.05)$. These results demonstrated that SAC/VAL plays a protective role against the vulnerability to AF during in vivo EPS.

\subsection{Sacubitril/valsartan inhibited Ang囚-induced Smad2/3, p38 and JNK activation}

To explain the mechanism of protective effects of SAC/VAL against Ang $₫$ induced atrial fibrosis, the relative protein expression of p-Smad2/3 and non-Smad proteins (ERK1/2, p-ERK1/2, p38 MAPK, p-p38

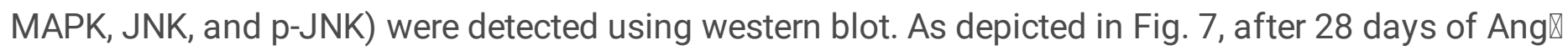
infusion, the relative expressions of p-Smad2/3, p-ERK1/2, p-p38 MAPK, and p-JNK were significantly increased in rats treated with saline. Both SAC/VAL and VAL could significantly reduce the expression of the abovementioned proteins. Compared with the saline group, SAC/VAL caused about a $27 \%$ decrease in the relative expression of $p-S m a d 2 / 3$, a $28 \%$ decrease in the relative expression of $p 38$, and a $25 \%$ decrease in the relative expression of $p$-JNK more than VAL $(p<0.05)$. However, no significant difference was observed between the relative expression of $p$-ERK in both SAC/VAL and VAL groups. The level of tERK1/2, t-p38, and t-JNK did not exhibit a marked difference among the three groups as well.

\section{Discussion}

The fibrous scar in atriums, acting as electrical barriers, uncouples the well-connected syncytium and increases heterogeneous electrical conduction[25]. This interaction between atrial structural remodeling and electrical remodeling allows for AF initiation and perpetuation [26]. However, as the most notable feature of atrial remodeling, atrial fibrosis mechanisms have not yet been fully elucidated. A growing body of evidence has suggested that atrial fibrosis might be a potential therapeutic target for AF[8-10]. In

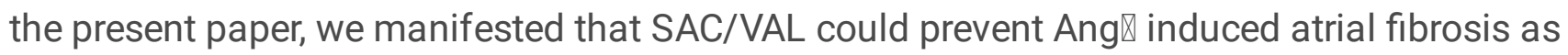
demonstrated by less distorted LA architecture, lower deposition of collagen $\nabla$ and $\nabla$, and attenuated distribution and expression of both a-SMA and vimentin. We also demonstrated that the susceptibility to atrial arrhythmias was significantly decreased following SAC/VAL treatment. We further verified that these protective roles of SAC/VAL could be attributable to the inhibition of p-Smad2/3, p-p38 MAPK, and p-JNK pathways.

Currently, therapeutic regimens, including ARBs, have been recommended for AF prevention by their capabilities to moderate the atrial structural and electrical remodeling through inhibition of RAAS in addition to their anti-hypertensive effects[27]. Likewise, active NPs have also been proven to inhibit fibrotic responses to prevent structural and electrical remodeling, as evidenced by the reduced risk of postoperative AF occurrence after administration of recombinant human atrial natriuretic peptides (ANP) or brain natriuretic peptides (BNP) $[28,29]$. The same effects can be achieved by inhibiting NEP to increase endogenous NPs[30, 31]. SAC/VAL is an agent with dual inhibitory effects on ACE and NEP. The latter enzyme is responsible for the degradation of three NPs - ANP, BNP, and C-type natriuretic peptide (CNP). All these three NPs, upon binding to their receptors, are capable of elevating the cGMP level and

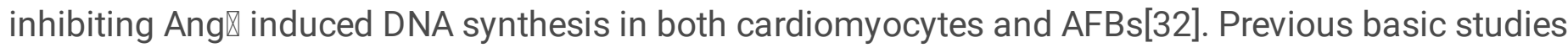
have substantively focused on the ventricle, and results from these studies shown that SAC/VAL has an inhibitory effect on ventricular fibrosis and thus preserves systolic cardiac function[21, 33, 34]. A recent 
study used the rabbit MI model demonstrated that SAC/VAL could also ameliorate post MI electrophysiologic remodeling and alleviate ventricular tachyarrhythmia inducibility. However, a few studies have been conducted to determine whether SAC/VAL could attenuate atrial fibrosis and decrease AF inducibility. In our study, we found that SAC/VAL did reduce AF susceptibility by inhibiting atrial fibrosis, which were consistent with two recent studies regarding the impact of SAC/VAL on AF[30, 31]. We also found that VAL had less evident effects on the prevention of atrial fibrosis and reduction of atrial arrhythmias inducibility than SAC/VAL. Previous study reported that simultaneous inhibition of NEP and

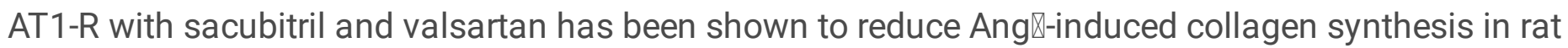
AFBs to a greater extent than either compound alone[33]. Moreover, we verified the ability of SAC/VAL to inhibit the proliferation, migration, and differentiation of atrial fibroblasts both in vivo and in vitro. We also observed that elevated ANP levels in the SAC/VAL group were accompanied by a significant reduction in the extent of atrial fibrosis as well as the proliferation, differentiation, and migration of AFBs. Indeed, endogenous ANP released by cardiomyocytes inhibited atrial fibroblasts proliferation and collagen deposition[35].

To date, the role of SAC/VAL in suppressing ventricular fibrosis is well-defined, but the mechanism behind it remains enigmatical by the current evidence, not to mention the mechanism underlying the inhibition of atrial fibrosis by SAC/VAL[20-22]. Only one study focusing atrial electrical remodeling demonstrated that SAC/VAL could reduce AF susceptibility by inhibiting the calcineurin/nuclear factor of activated T cell (NFAT) pathway[30]. In our study, we finally looked at signaling pathways to give our answer as to how SAC/VAL exerts its anti-fibrosis effects in atriums. Atrial fibrosis necessitates involving RAAS, a crucial

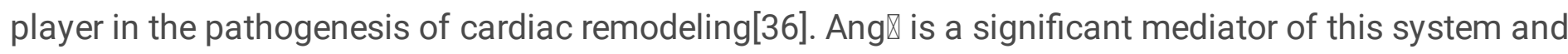
renders manifold downstream cytokines active through multiple signaling pathways, including the mitogen-activated protein kinase (MAPK) pathway. In addition to directly contributing to fibrosis, activation of the MAPK signaling pathway stimulates the secretion of TGF- $\beta 1$, while TGF- $\beta 1$ has also been reported to implicate in the development of atrial fibrosis, which promotes the secretion and differentiation of atrial fibroblasts via the typical Smad-dependent pathway and noncanonical Smadindependent pathways[37]. A previous study has suggested that SAC/VAL possesses the capacity to

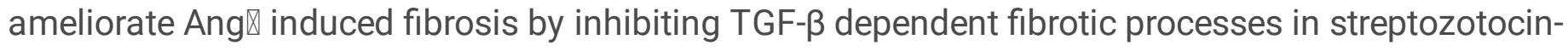
induced diabetic mice model[34]. Hence, to clarify the mechanisms of the protective effects of SAC/VAL, we detected the relative expression of Smad proteins and MAPK proteins. In our study, we observed that

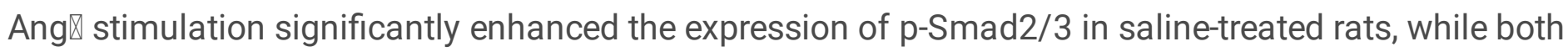
VAL and SAC/VAL treatment reduced the level of $p-S m a d 2 / 3$. When comparing the latter two groups, it was found that SAC/VAL reduced the expression of $\mathrm{p}-\mathrm{Smad} 2 / 3$ more significantly. The results suggested that decreasing the phosphorylation of Smad2/3 may the mechanism by which SAC/VAL exerts its role in antagonizing Ang $₫$-induced fibrosis. In addition to the above canonical pathways, previous studies have demonstrated that SAC/VAL could inhibit the phosphorylation of JNK and p38MAPK in experimental models of diabetic cardiomyopathy[38]. Our study also found the pronouncedly high level of $p$-JNK, $p$ -

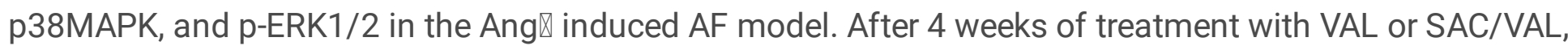
these proteins' expression levels declined dramatically. Moreover, the expression levels of $p$-JNK and $p$ - 
p38MAPK, not p-ERK1/2, were significantly lower in the SAC/VAL group compared to the VAL group, which may explain the reason for the superiority of SAC/VAL over VAL in attenuating atrial fibrosis and reducing AF inducibility.

\section{Conclusion}

In summary, our study revealed part of the mechanisms by which SAC/VAL inhibits atrial fibrosis and decreases AF inducibility, which is beneficial for enriching AF treatment regimens. We also demonstrated that SAC/VAL is superior to VAL in reducing atrial structural remodeling and attenuated AF susceptibility, possibly in part through inhibition of the p-Smad2/3, p-p38 MAPK, and p-JNK pathways.

\section{Abbreviations}




\begin{tabular}{|c|c|}
\hline a-SMA & a-smooth muscle actin \\
\hline SAC/VAL & Sacubitril/valsartan \\
\hline AT-R & Angiotensin receptor \\
\hline ACEls & Angiotensin-converting enzyme inhibitors \\
\hline AF & Atrial fibrillation \\
\hline AFB & Atrial fibroblast \\
\hline Ang $\rrbracket$ & Angiotensin $\otimes$ \\
\hline ANP & Atrial natriuretic peptide \\
\hline ARBs & Angiotensin receptor blockers \\
\hline BNP & B-type natriuretic peptide \\
\hline CNP & C-type natriuretic peptide \\
\hline DBP & Diastolic blood pressure \\
\hline EF & Ejection fraction \\
\hline ELISA & Enzyme-linked immunosorbent assay \\
\hline EPS & Electrophysiological study \\
\hline ERK & Extracellular signal-regulated kinase \\
\hline HFrEF & Heart failure with reduced ejection fraction \\
\hline $\mathrm{IHC}$ & Immunohistochemistry \\
\hline JNK & C-Jun N-terminal kinase \\
\hline LAD & Left atrial diameter \\
\hline LVDD & LV end-diastolic diameter \\
\hline MAPK & Mitogen-activated protein kinase \\
\hline MBP & Mean blood pressure \\
\hline MI & Myocardial infarction \\
\hline MTT & 3-(4,5-dimethylthiazol-2-yl)-2,5-diphenyltetrazolium Bromide \\
\hline MV E/A & Mitral valvular early and atrial peaks \\
\hline NEP & Neutral endopeptidase \\
\hline NFAT & Calcineurin/nuclear factor of activated T cell \\
\hline NF-KB & Nuclear factor kappa-light-chain-enhancer of activated B cells \\
\hline
\end{tabular}


NLRP3 Ucleotide-binding oligomerization domain-like receptor protein 3

NPs Natriuretic peptides

NT-proBNP N-terminal pro-brain natriuretic peptide

p-CaMKII Phosphorylated expression calmodulin-dependent protein kinase II

PKG Protein kinase G

RAAS Renin-angiotensin-aldosterone system

SBP Systolic blood pressure

Smad Small mother against decapentaplegic

\section{Declarations}

\subsection{Ethics approval and consent to participate}

All experimental procedures were conducted in compliance with both the Animal Care and Use Committee of Capital Medical University and the Guide for the Care and Use of Laboratory Animals published by the National Institutes of Health (the 8th Edition, NRC 2011). This article does not contain any studies with human participants performed by any of the authors.

\subsection{Consent for publication}

Not applicable.

\subsection{Availability of data and materials}

Not applicable.

\subsection{Competing interests}

The authors declare that they have no conflicts of interest.

\subsection{Funding}

This study was supported by Grants from the National Natural Science Foundation of China (Nos. 81700271), S\&T Program of Hebei (Nos. H2018105054).

\subsection{Authors' contributions}

Chang-yi Li, Song-nan Li and Lei Zhao conceived and planned the experiments. Song-nan Li, Jing-rui Zhang, and Hui Xi carried out the experiments. Song-nan Li, Chang-yi Li, and Lei Zhao contributed to the interpretation of the results. Song-nan Li and Jing-rui Zhang took the lead in writing the manuscript. All authors have read and approved the final manuscript. 


\subsection{Acknowledgements}

Not applicable.

\section{References}

1. Staerk, L.; Sherer, J. A.; Ko, D.; Benjamin, E. J.; Helm, R. H., Atrial Fibrillation: Epidemiology, Pathophysiology, and Clinical Outcomes. Circulation research 2017, 120, 1501-1517.

2. Christiansen, C. B.; Gerds, T. A.; Olesen, J. B.; Kristensen, S. L.; Lamberts, M.; Lip, G. Y.; Gislason, G. H.; Køber, L.; Torp-Pedersen, C., Atrial fibrillation and risk of stroke: a nationwide cohort study. Europace 2016, 18, 1689-1697.

3. Holmqvist, F.; Kesek, M.; Englund, A.; Blomström-Lundqvist, C.; Karlsson, L. O.; Kennebäck, G.; Poçi, D.; Samo-Ayou, R.; Sigurjónsdóttir, R.; Ringborn, M.; Herczku, C.; Carlson, J.; Fengsrud, E.; Tabrizi, F.; Höglund, N.; Lönnerholm, S.; Kongstad, O.; Jönsson, A.; Insulander, P., A decade of catheter ablation of cardiac arrhythmias in Sweden: ablation practices and outcomes. European heart journal 2019, 40, 820-830.

4. Wijesurendra, R. S.; Casadei, B., Mechanisms of atrial fibrillation. Heart (British Cardiac Society) 2019, $105,1860-1867$.

5. Chen, P. S.; Chen, L. S.; Fishbein, M. C.; Lin, S. F.; Nattel, S., Role of the autonomic nervous system in atrial fibrillation: pathophysiology and therapy. Circulation research 2014, 114, 1500-1515.

6. Pellman, J.; Sheikh, F., Atrial fibrillation: mechanisms, therapeutics, and future directions. Compr Physiol 2015, 5, 649-665.

7. Dzeshka, M. S.; Lip, G. Y.; Snezhitskiy, V.; Shantsila, E., Cardiac Fibrosis in Patients With Atrial Fibrillation: Mechanisms and Clinical Implications. Journal of the American College of Cardiology 2015, 66, 943-959.

8. Ma, J.; Ma, S.; Yin, C.; Wu, H., Matrine reduces susceptibility to postinfarct atrial fibrillation in rats due to antifibrotic properties. J Cardiovasc Electrophysio/ 2018, 29, 616-627.

9. Liu, M.; Li, W.; Wang, H.; Yin, L.; Ye, B.; Tang, Y.; Huang, C., CTRP9 Ameliorates Atrial Inflammation, Fibrosis, and Vulnerability to Atrial Fibrillation in Post-Myocardial Infarction Rats. Journal of the American Heart Association 2019, 8, e013133.

10. Beiert, T.; Tiyerili, V.; Knappe, V.; Effelsberg, V.; Linhart, M.; Stöckigt, F.; Klein, S.; Schierwagen, R.; Trebicka, J.; Nickenig, G.; Schrickel, J. W.; Andrié, R. P., Relaxin reduces susceptibility to post-infarct atrial fibrillation in mice due to anti-fibrotic and anti-inflammatory properties. Biochem Biophys Res Commun 2017, 490, 643-649.

11. Ge, Z.; Chen, Y.; Wang, B.; Zhang, X.; Yan, Y.; Zhou, L.; Zhang, Y.; Xie, Y., MFGE8 attenuates Ang-IIinduced atrial fibrosis and vulnerability to atrial fibrillation through inhibition of TGF- $\beta 1 / \mathrm{Smad} 2 / 3$ pathway. Journal of molecular and cellular cardiology 2020, 139, 164-175.

12. Ehrlich, J. R.; Hohnloser, S. H.; Nattel, S., Role of angiotensin system and effects of its inhibition in atrial fibrillation: clinical and experimental evidence. European heart journa/ 2006, 27, 512-518. 
13. Nattel, S., Molecular and Cellular Mechanisms of Atrial Fibrosis in Atrial Fibrillation. JACC Clin Electrophysio/ 2017, 3, 425-435.

14. Jansen, H. J.; Mackasey, M.; Moghtadaei, M.; Liu, Y.; Kaur, J.; Egom, E. E.; Tuomi, J. M.; Rafferty, S. A.; Kirkby, A. W.; Rose, R. A., NPR-C (Natriuretic Peptide Receptor-C) Modulates the Progression of Angiotensin II-Mediated Atrial Fibrillation and Atrial Remodeling in Mice. Circ Arrhythm Electrophysiol 2019, 12, e006863.

15. Okazaki, H.; Minamino, T.; Tsukamoto, O.; Kim, J.; Okada, K.; Myoishi, M.; Wakeno, M.; Takashima, S.; Mochizuki, N.; Kitakaze, M., Angiotensin II type 1 receptor blocker prevents atrial structural remodeling in rats with hypertension induced by chronic nitric oxide inhibition. Hypertension research : official journal of the Japanese Society of Hypertension 2006, 29, 277-284.

16. Li, D.; Shinagawa, K.; Pang, L.; Leung, T. K.; Cardin, S.; Wang, Z.; Nattel, S., Effects of angiotensinconverting enzyme inhibition on the development of the atrial fibrillation substrate in dogs with ventricular tachypacing-induced congestive heart failure. Circulation 2001, 104, 2608-2614.

17. Kaplinsky, E., Sacubitril/valsartan in heart failure: latest evidence and place in therapy. Therapeutic advances in chronic disease 2016, 7, 278-290.

18. McMurray, J. J.; Packer, M.; Desai, A. S.; Gong, J.; Lefkowitz, M. P.; Rizkala, A. R.; Rouleau, J. L.; Shi, V. C.; Solomon, S. D.; Swedberg, K.; Zile, M. R., Angiotensin-neprilysin inhibition versus enalapril in heart failure. The New England journal of medicine 2014, 371, 993-1004.

19. Chang, P. C.; Wo, H. T.; Lee, H. L.; Lin, S. F.; Chu, Y.; Wen, M. S.; Chou, C. C., Sacubitril/Valsartan Therapy Ameliorates Ventricular Tachyarrhythmia Inducibility in a Rabbit Myocardial Infarction Model. Journal of cardiac failure 2020, 26, 527-537.

20. Vaskova, E.; Ikeda, G.; Tada, Y.; Wahlquist, C.; Mercola, M.; Yang, P. C., Sacubitril/Valsartan Improves Cardiac Function and Decreases Myocardial Fibrosis Via Downregulation of Exosomal miR-181a in a Rodent Chronic Myocardial Infarction Model. Journal of the American Heart Association 2020, 9, e015640.

21. Burke, R. M.; Lighthouse, J. K.; Mickelsen, D. M.; Small, E. M., Sacubitril/Valsartan Decreases Cardiac Fibrosis in Left Ventricle Pressure Overload by Restoring PKG Signaling in Cardiac Fibroblasts. Circulation. Heart failure 2019, 12, e005565.

22. Li, X.; Zhu, Q.; Wang, Q.; Zhang, Q.; Zheng, Y.; Wang, L.; Jin, Q., Protection of Sacubitril/Valsartan against Pathological Cardiac Remodeling by Inhibiting the NLRP3 Inflammasome after Relief of Pressure Overload in Mice. Cardiovascular drugs and therapy 2020, 34, 629-640.

23. Wang, W. W.; Zhang, F. L.; Chen, J. H.; Chen, X. H.; Fu, F. Y.; Tang, M. R.; Chen, L. L., Telmisartan reduces atrial arrhythmia susceptibility through the regulation of RAS-ERK and PI3K-Akt-eNOS pathways in spontaneously hypertensive rats. Can J Physiol Pharmacol 2015, 93, 657-665.

24. Wang, Q.; Yu, Y.; Zhang, P.; Chen, Y.; Li, C.; Chen, J.; Wang, Y.; Li, Y., The crucial role of activin A/ALK4 pathway in the pathogenesis of Ang-Il-induced atrial fibrosis and vulnerability to atrial fibrillation. Basic Res Cardiol 2017, 112, 47. 
25. Rog-Zielinska, E. A.; Norris, R. A.; Kohl, P.; Markwald, R., The Living Scar-Cardiac Fibroblasts and the Injured Heart. Trends Mol Med 2016, 22, 99-114.

26. Nguyen, T. P.; Qu, Z.; Weiss, J. N., Cardiac fibrosis and arrhythmogenesis: the road to repair is paved with perils. Journal of molecular and cellular cardiology 2014, 70, 83-91.

27. Sestito, A., Hypertension therapy and cardiovascular protection. Effects of angiotensin II receptor block with Valsartan. Eur Rev Med Pharmacol Sci 2011, 15, 1247-1255.

28. Sezai, A.; lida, M.; Yoshitake, I.; Wakui, S.; Osaka, S.; Kimura, H.; Yaoita, H.; Hata, H.; Shiono, M.; Nakai, T.; Takayama, T.; Kunimoto, S.; Kasamaki, Y.; Hirayama, A., Carperitide and atrial fibrillation after coronary bypass grafting: the Nihon University working group study of low-dose HANP infusion therapy during cardiac surgery trial for postoperative atrial fibrillation. Circ Arrhythm Electrophysiol 2015, 8, 546-553.

29. Mentzer, R. M., Jr.; Oz, M. C.; Sladen, R. N.; Graeve, A. H.; Hebeler, R. F., Jr.; Luber, J. M., Jr.; Smedira, N. G., Effects of perioperative nesiritide in patients with left ventricular dysfunction undergoing cardiac surgery:the NAPA Trial. Journal of the American College of Cardiology 2007, 49, 716-726.

30. Li, L. Y.; Lou, Q.; Liu, G. Z.; Lv, J. C.; Yun, F. X.; Li, T. K.; Yang, W.; Zhao, H. Y.; Zhang, L.; Bai, N.; Zhan, C. C.; Yu, J.; Zang, Y. X.; Li, W. M., Sacubitril/valsartan attenuates atrial electrical and structural remodelling in a rabbit model of atrial fibrillation. European journal of pharmacology 2020, 881 , 173120.

31. Suo, Y.; Yuan, M.; Li, H.; Zhang, Y.; Li, Y.; Fu, H.; Han, F.; Ma, C.; Wang, Y.; Bao, Q.; Li, G., Sacubitril/Valsartan Improves Left Atrial and Left Atrial Appendage Function in Patients With Atrial Fibrillation and in Pressure Overload-Induced Mice. Front Pharmacol 2019, 10, 1285.

32. Fujisaki, H.; Ito, H.; Hirata, Y.; Tanaka, M.; Hata, M.; Lin, M.; Adachi, S.; Akimoto, H.; Marumo, F.; Hiroe, M., Natriuretic peptides inhibit angiotensin II-induced proliferation of rat cardiac fibroblasts by blocking endothelin-1 gene expression. J Clin Invest 1995, 96, 1059-1065.

33. von Lueder, T. G.; Wang, B. H.; Kompa, A. R.; Huang, L.; Webb, R.; Jordaan, P.; Atar, D.; Krum, H., Angiotensin receptor neprilysin inhibitor LCZ696 attenuates cardiac remodeling and dysfunction after myocardial infarction by reducing cardiac fibrosis and hypertrophy. Circulation. Heart failure 2015, 8, 71-78.

34. Suematsu, Y.; Miura, S.; Goto, M.; Matsuo, Y.; Arimura, T.; Kuwano, T.; Imaizumi, S.; Iwata, A.; Yahiro, E.; Saku, K., LCZ696, an angiotensin receptor-neprilysin inhibitor, improves cardiac function with the attenuation of fibrosis in heart failure with reduced ejection fraction in streptozotocin-induced diabetic mice. European journal of heart failure 2016, 18, 386-393.

35. Maki, T.; Horio, T.; Yoshihara, F.; Suga, S.; Takeo, S.; Matsuo, H.; Kangawa, K., Effect of neutral endopeptidase inhibitor on endogenous atrial natriuretic peptide as a paracrine factor in cultured cardiac fibroblasts. British journal of pharmacology 2000, 131, 1204-1210.

36. Kirchhof, P.; Fabritz, L., Of hammers and screws: renin-angiotensin-aldosterone system inhibition to prevent atrial fibrillation in patients with hypertension. European heart journal 2014, 35, 1169-1171. 
37. Biernacka, A.; Dobaczewski, M.; Frangogiannis, N. G., TGF- $\beta$ signaling in fibrosis. Growth Factors 2011, 29, 196-202.

38. Ge, Q.; Zhao, L.; Ren, X. M.; Ye, P.; Hu, Z. Y., Feature article: LCZ696, an angiotensin receptor-neprilysin inhibitor, ameliorates diabetic cardiomyopathy by inhibiting inflammation, oxidative stress and apoptosis. Experimental biology and medicine (Maywood, N.J.) 2019, 244, 1028-1039.

\section{Figures}




\section{Saline}

A
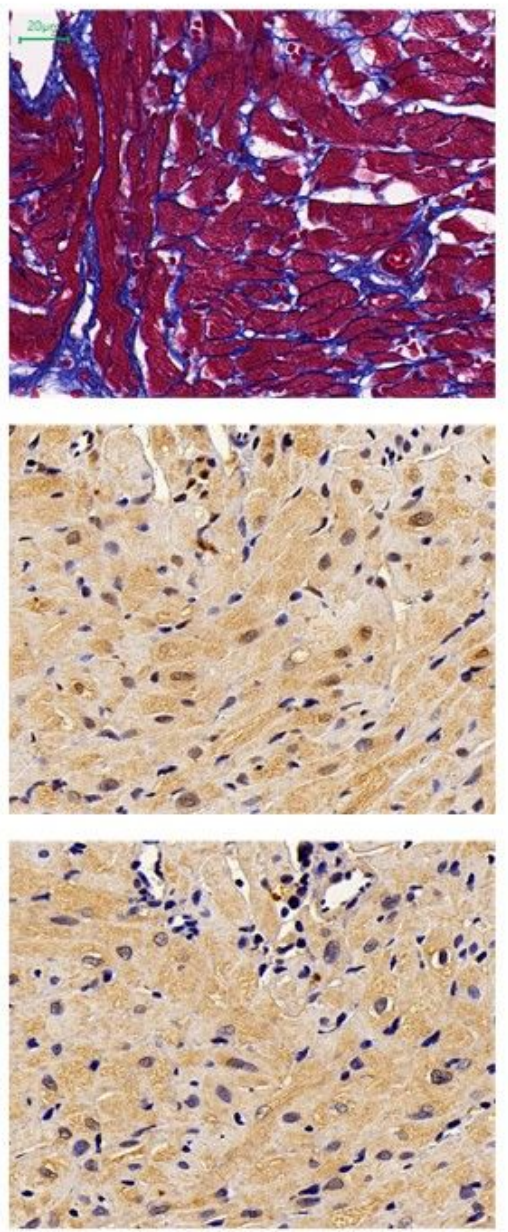

B

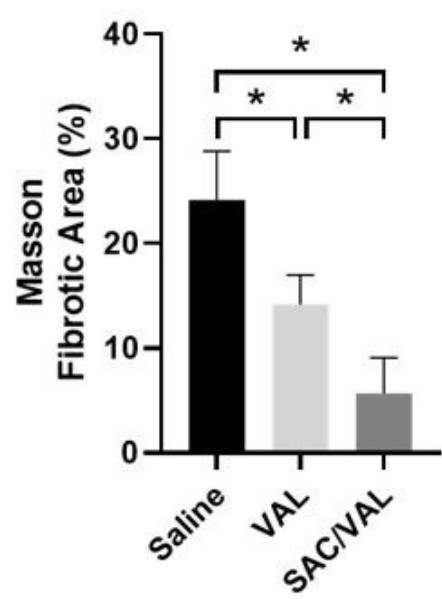

VAL
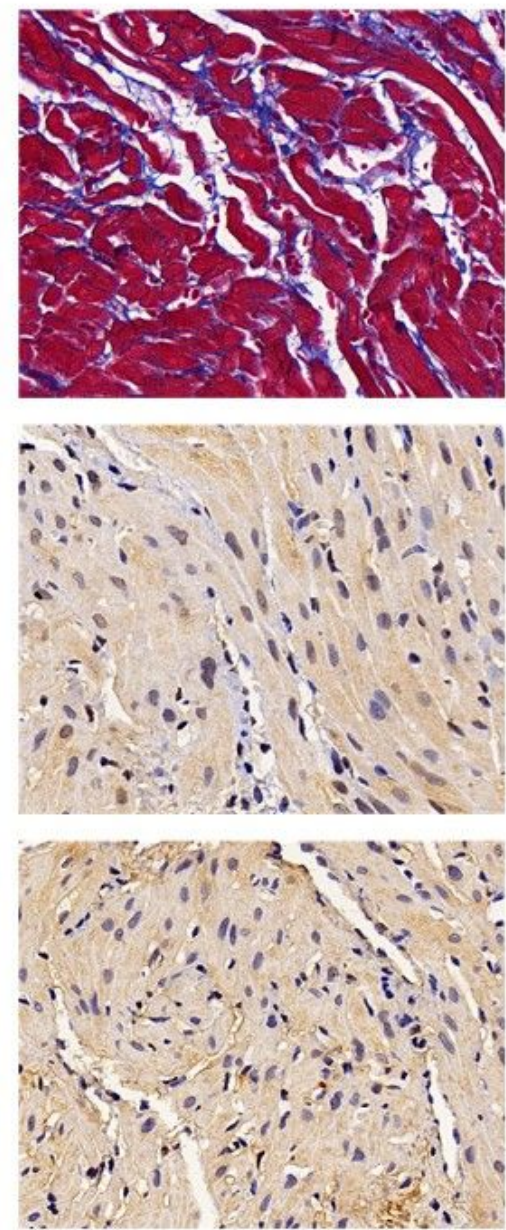

C

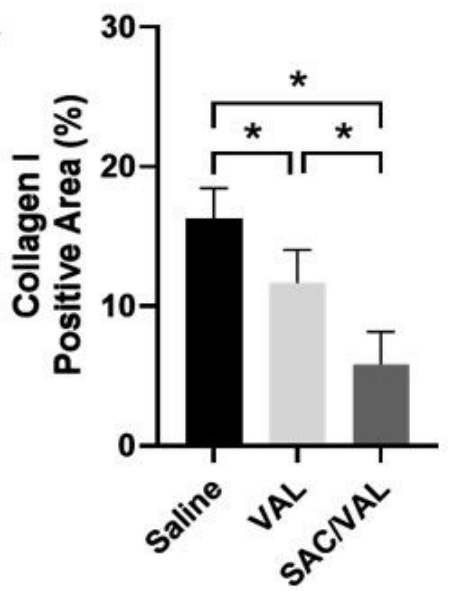

SAC/VAL
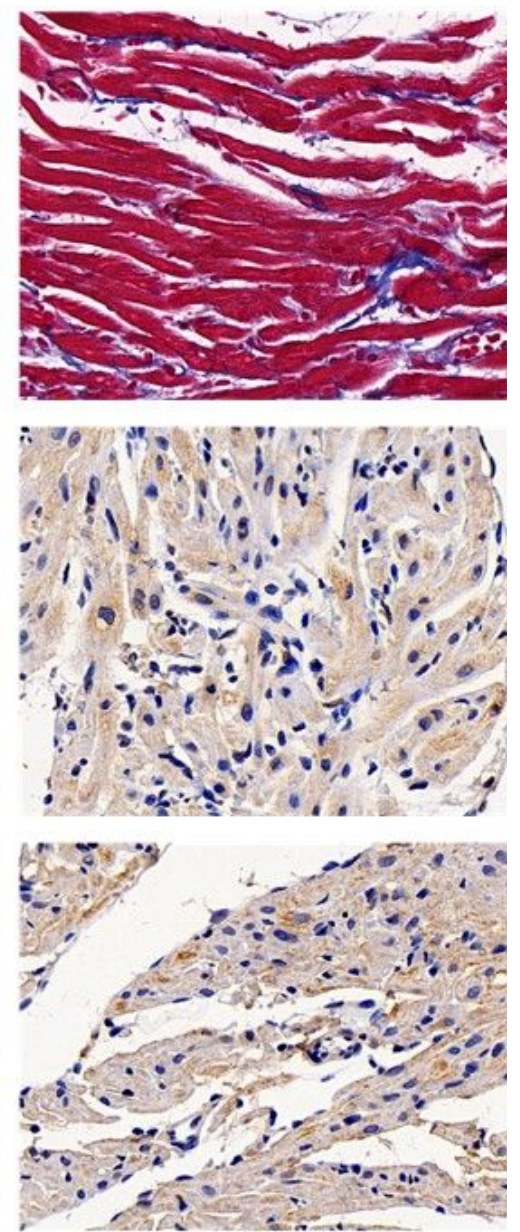

D

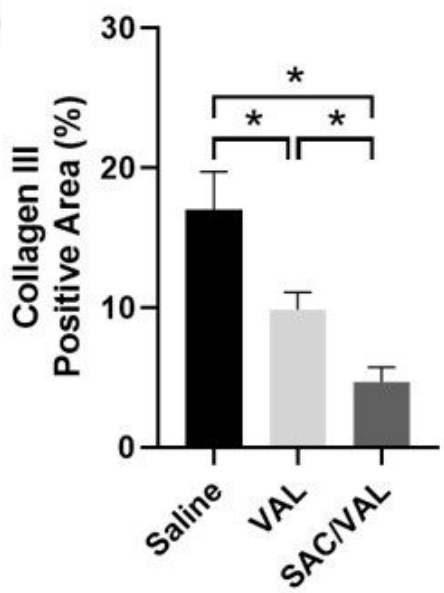

Figure 1

Sacubitril-valsartan mitigates Ang-II-induced atrial fibrosis in vivo. (A) Representative histological images of the Masson trichrome staining (upper panel), of the immunohistochemistry staining of collagen I (middle panel) and collagen III (lower panel) of the atrial tissues after 28-day Ang-ll infusion. (B, C and D) Quantitative analysis of the stained fibrotic areas of the atrial tissues (right), the collagen I 
(middle) and the collagen III (left). Data are expressed as the mean \pm SD ( $n=6$ samples per group). *P $<$ 0.05 vs. another group. VAL: Valsartan; SAC/VAL: Sacubitril-valsartan.

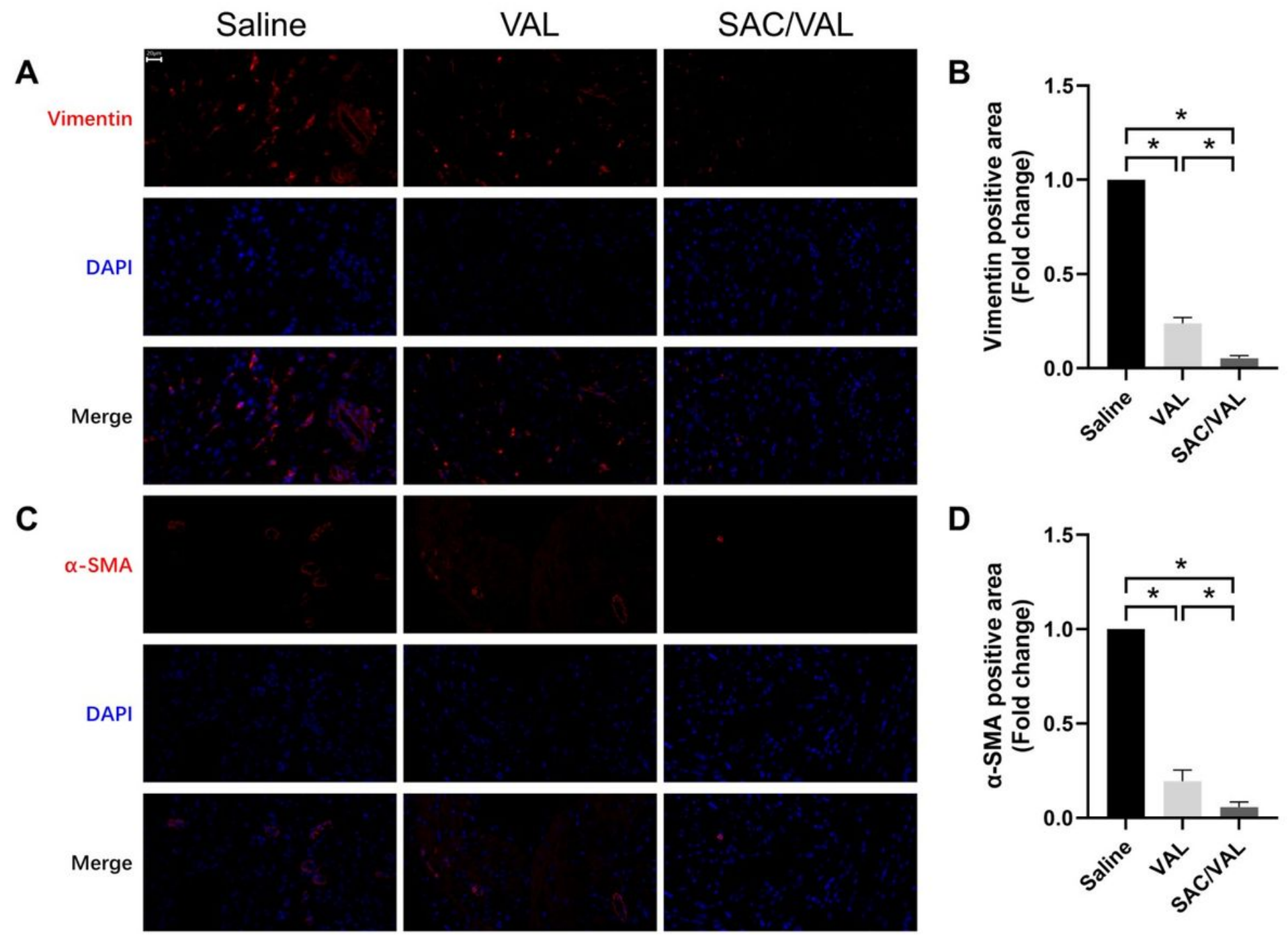

Figure 2

Effect of sacubitril-valsartan on the proliferation and differentiation of rat atriums. (A and C) Immunofluorescence staining of vimentin and a-SMA in atrial tissues post 28-day Ang-II administration. Vimentin and a-SMA were stained with Cy5 (red), nuclei were stained with DAPI (blue). (B and D) Quantification of each group shown in panel $A$ and C. Data are expressed as the mean $\pm S D(n=6$ samples per group). ${ }^{\star} \mathrm{P}<0.05$ vs another group. a-SMA: a-smooth muscle actin. VAL: Valsartan; SAC/VAL: Sacubitril-valsartan. 


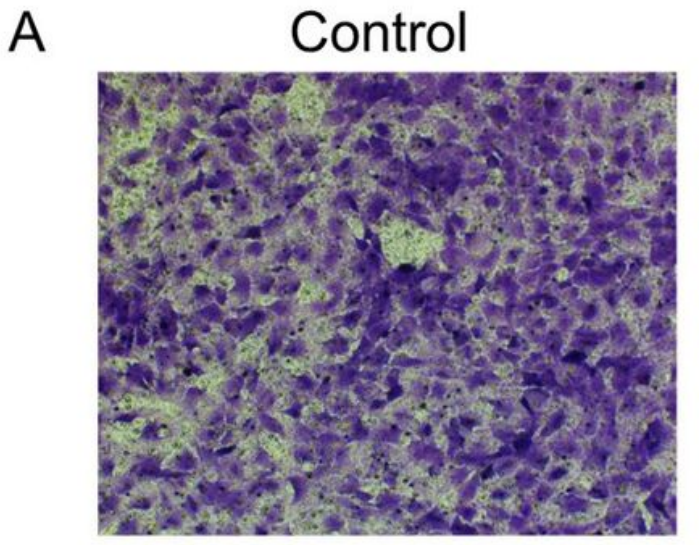

B

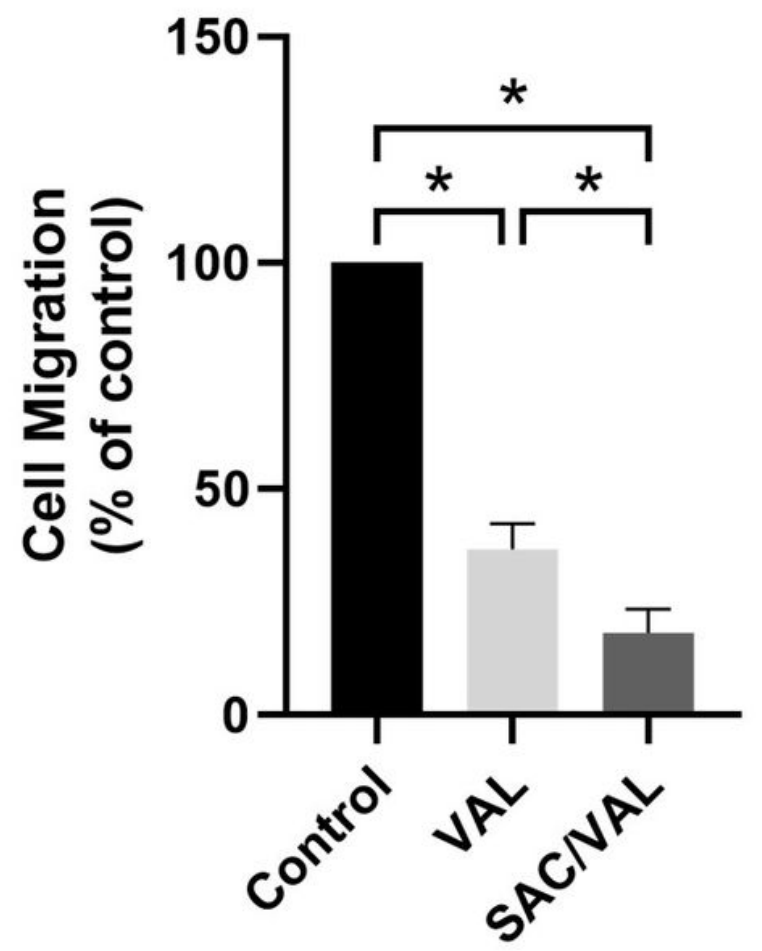

VAL

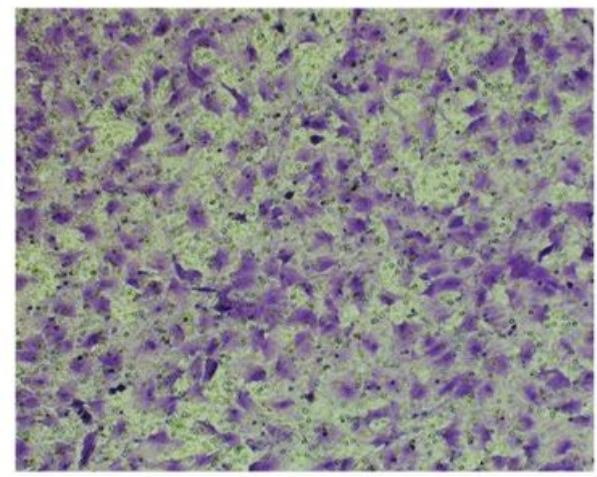

C
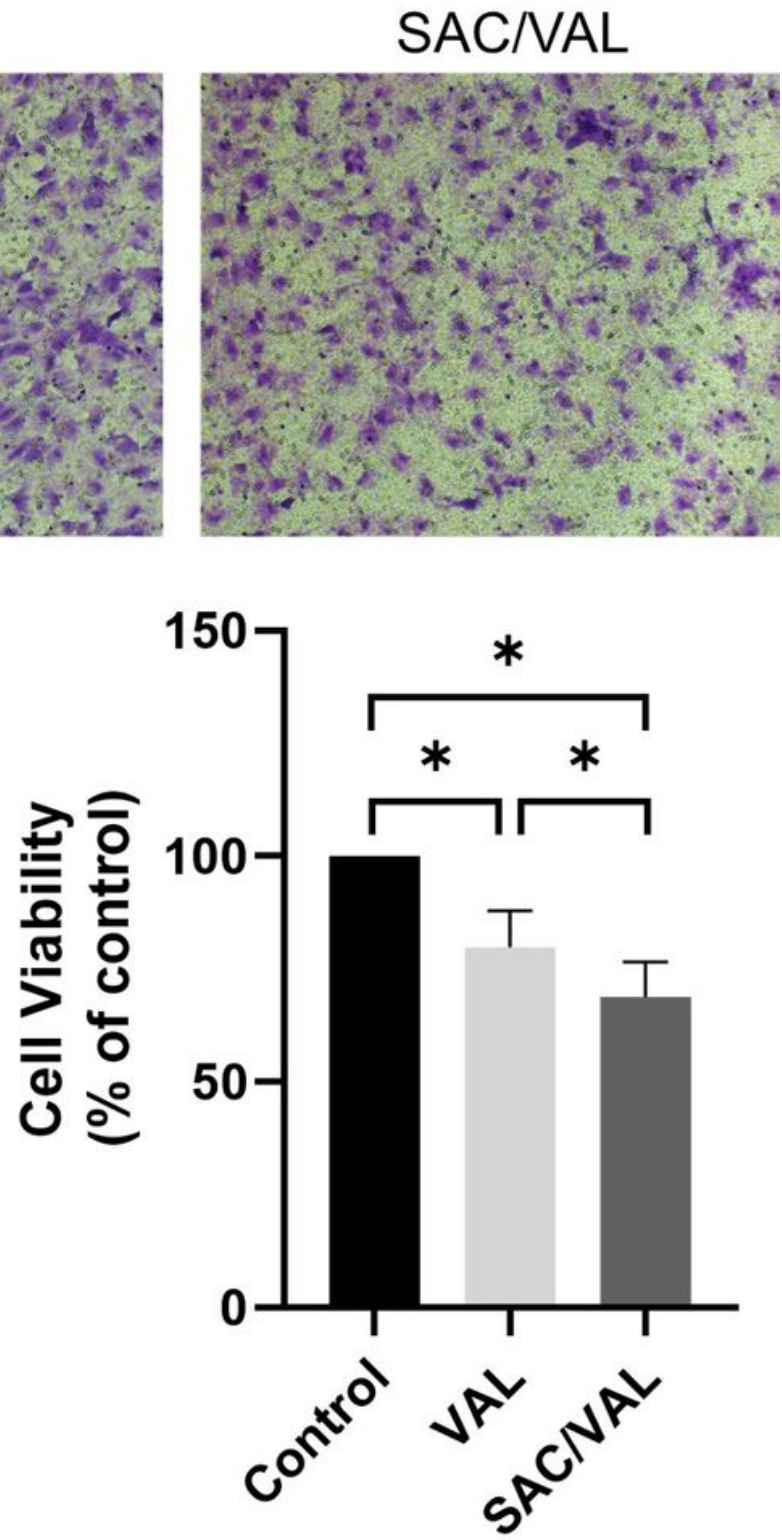

Figure 3

Sacubitril-valsartan decreases Ang囚-induced atrial fibroblasts migration and proliferation. (A) Representative images of fibroblasts from each experimental group penetrating an artificial basement membrane (×200 magnification). (B) Quantitative analysis of fibroblasts migration for each group shown in panel $A$ ( $n=5$ batches per group). (C) Effects of sacubitril-valsartan on proliferation of atrial fibroblasts determined by MTT assay ( $n=5$ batches per group). Ang-Il stimulated proliferation of atrial fibroblasts, but this effect was suppressed by sacubitril-valsartan administration. ${ }^{*} \mathrm{P}<0.05$ vs another group. VAL: Valsartan; SAC/VAL: Sacubitril-valsartan. 

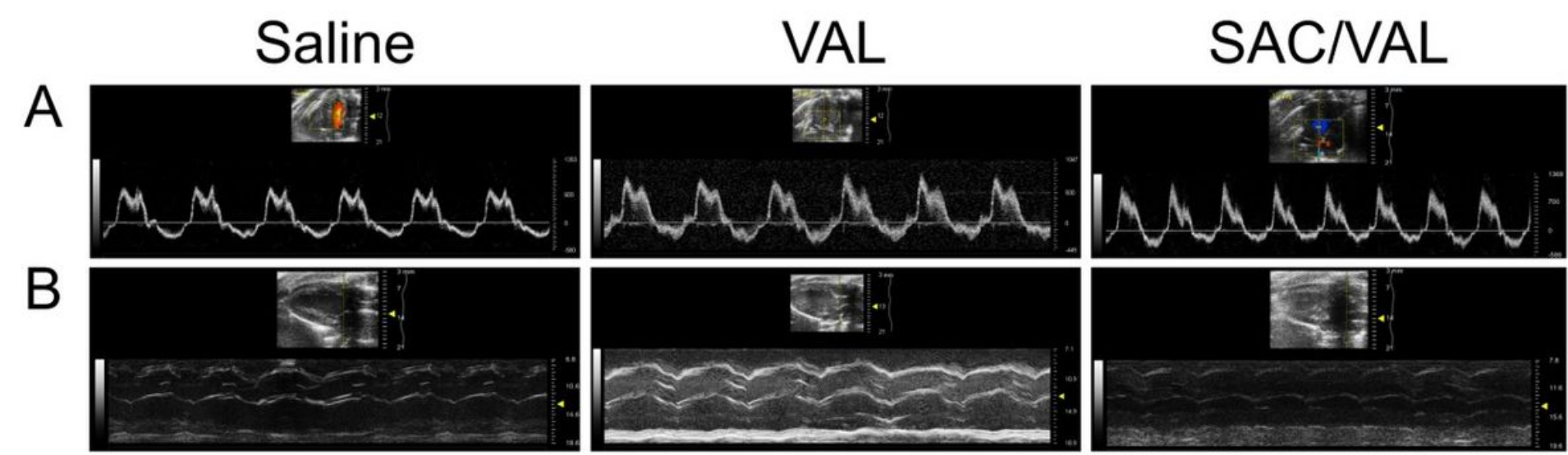

C
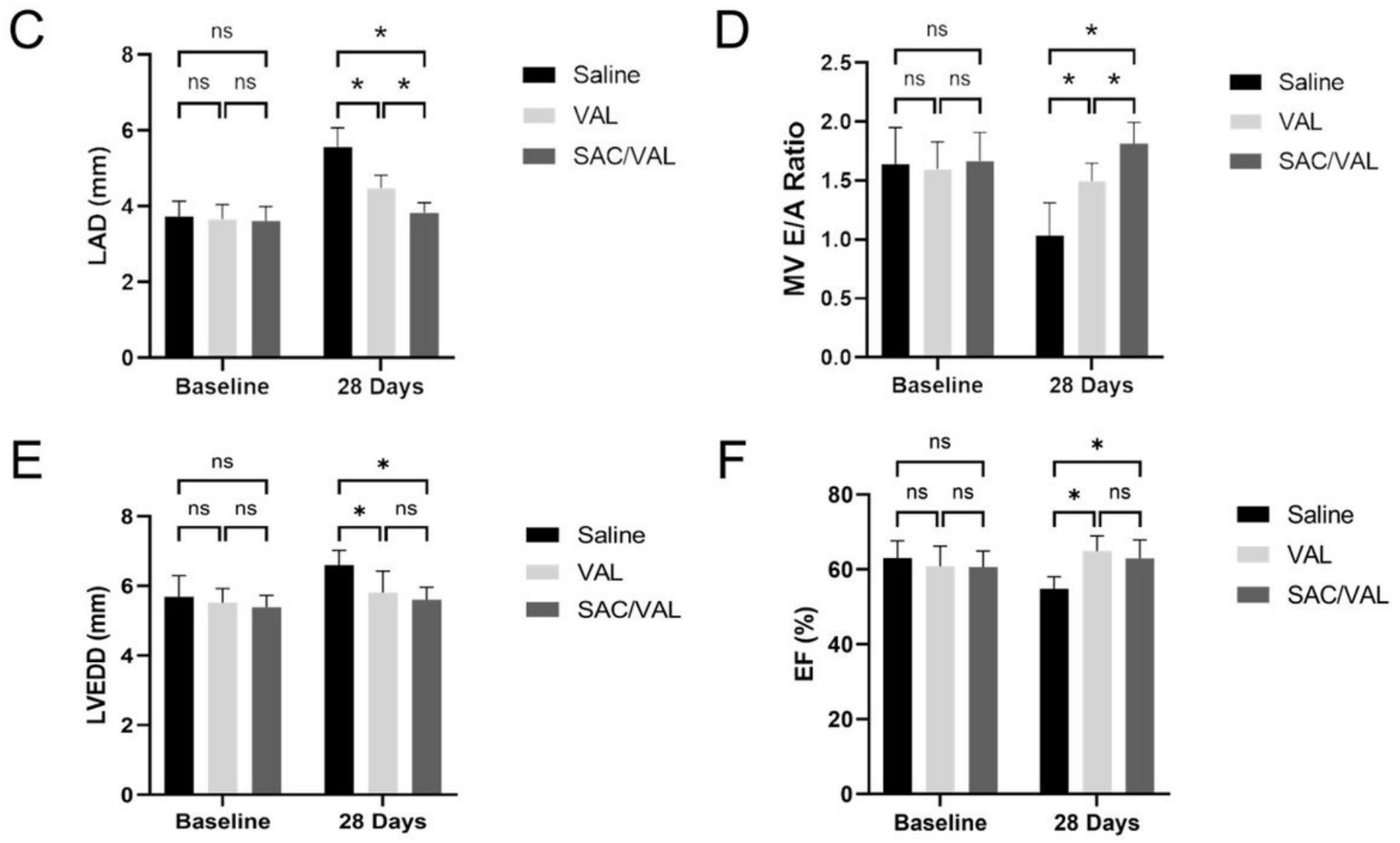

Figure 4

Sacubitril-valsartan improves heart functions and decreases blood pressure following Ang-Il-infusion. (A and $B)$ Representative in vivo images of the mitral valvular E/A ratio and left atrial dimensions after 28day Ang-II infusion. (C-F) LA, MV E/A, LVEDD, and EF were measured in saline-, valsartan-, and sacubitrilvalsartan-treated groups at 4 weeks after Ang-II infusion ( $n=6$ rats per group). ${ }^{*} P<0.05$ vs. another group; ns, not significant. LAD: Left atrial diameter; MV E/A: Mitral valvular early peak/atrial peak ratio; LVEDD, Left ventricular end diastolic diameter; EF: Ejection fraction. VAL: Valsartan; SAC/VAL: Sacubitrilvalsartan. 
A

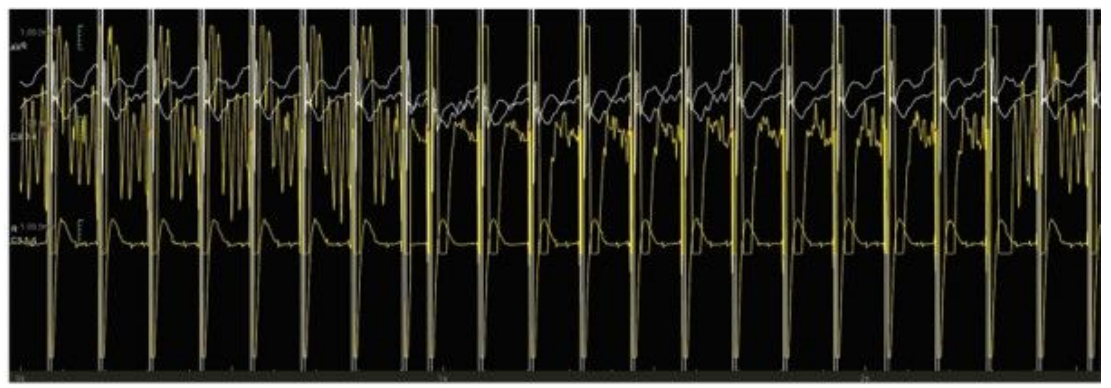

$\mathrm{B}$

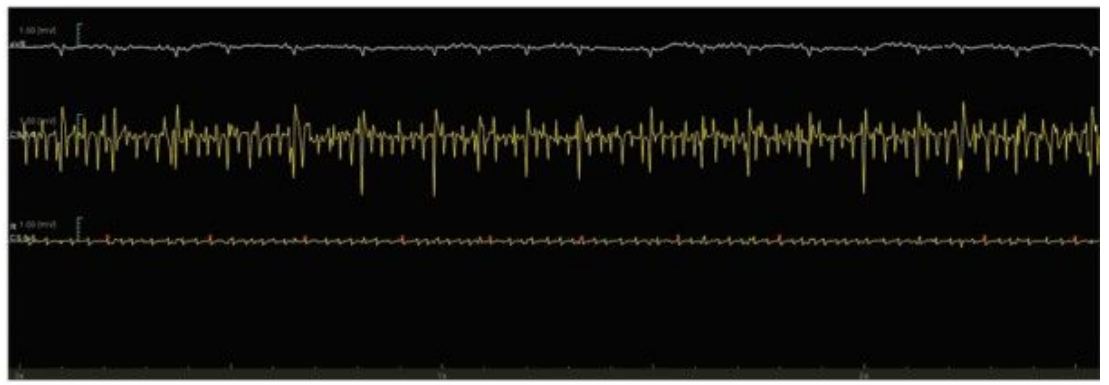

C
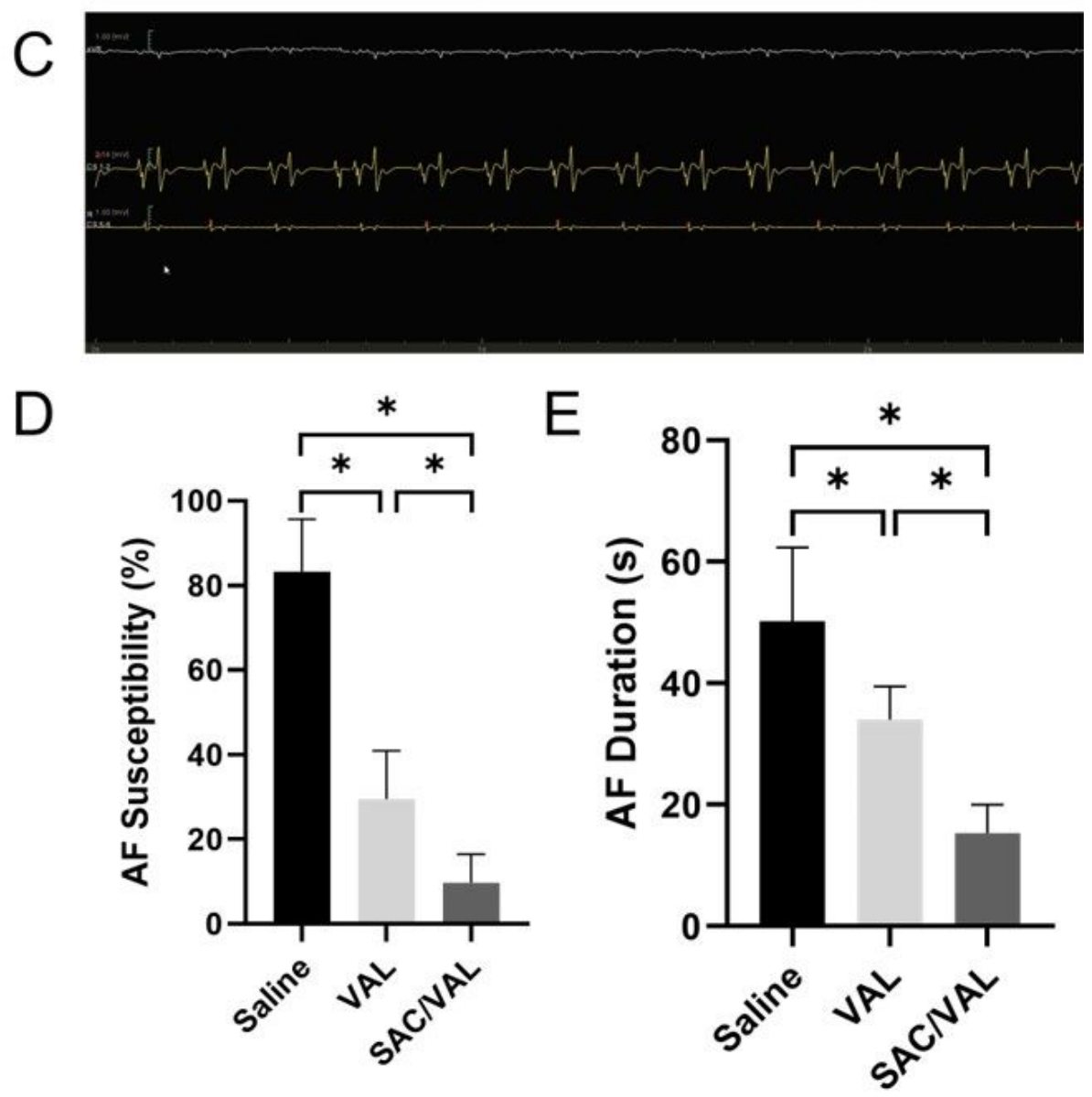

Figure 5

Plasma parameters were detected by ELISA assay. (A-C) Quantitative analysis of Ang-II (left), ANP (middle) and NT-proBNP (right) levels in plasma samples collected at 4 weeks after Ang-II infusion ( $n=6$ samples per group). (G-I) SBP, DBP and MBP were recorded at 28 days post-Ang-II administration. Data are expressed as the mean $\pm S D$ ( $n=6$ rats per group). ${ }^{*} P<0.05$ vs another group; $n s$, not significant. AngII: Angiotensin II; ANP: Atrial natriuretic peptide; NT-proBNP: N-terminal pro-brain natriuretic peptide; SBP, 
systolic blood pressure; MBP, mean blood pressure; DBP, diastolic blood pressure. VAL: Valsartan; SAC/VAL: Sacubitril-valsartan.

A

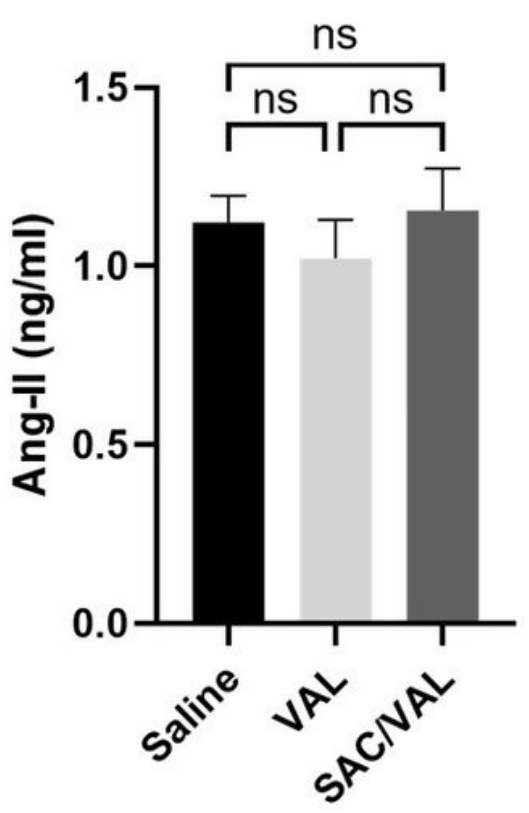

$\mathrm{D}$

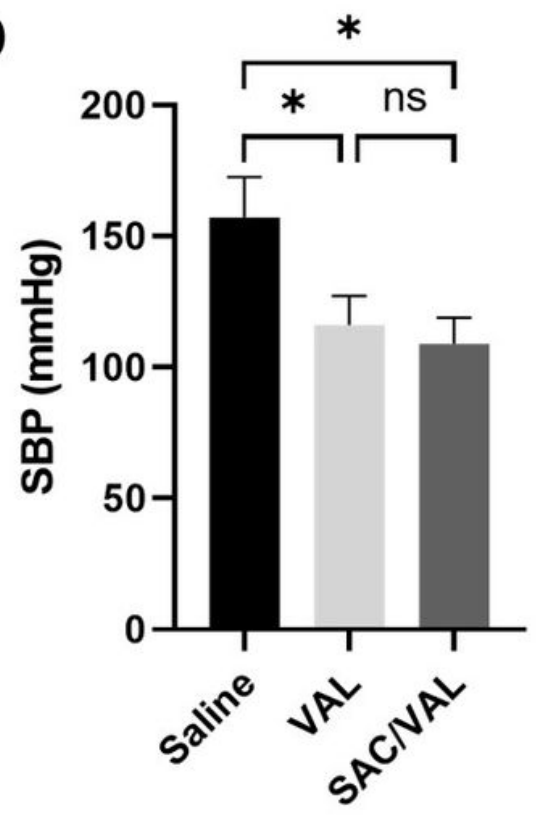

B

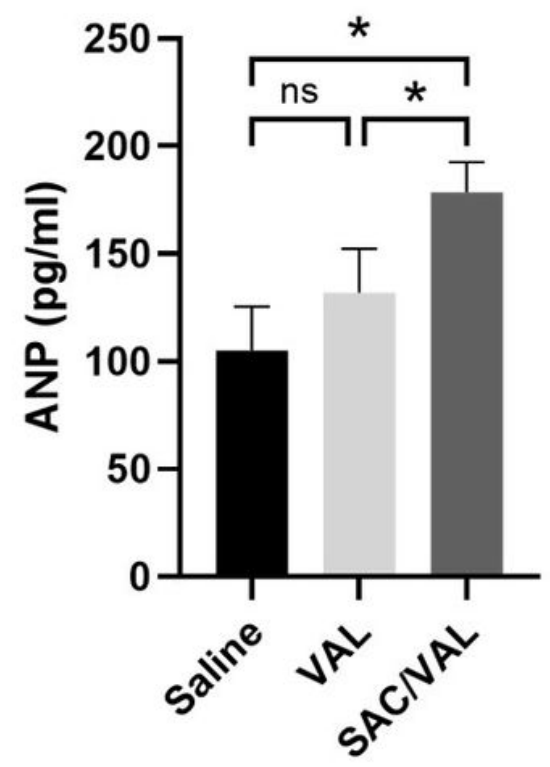

$E$

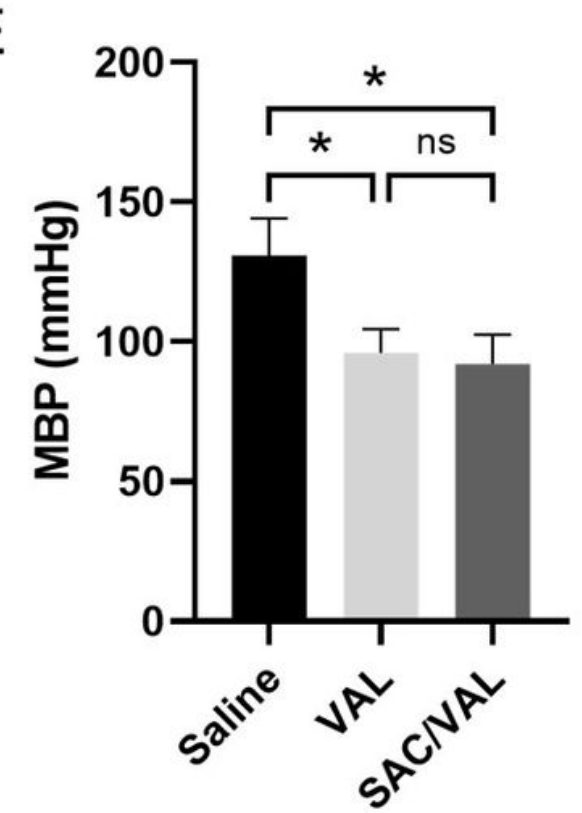

C

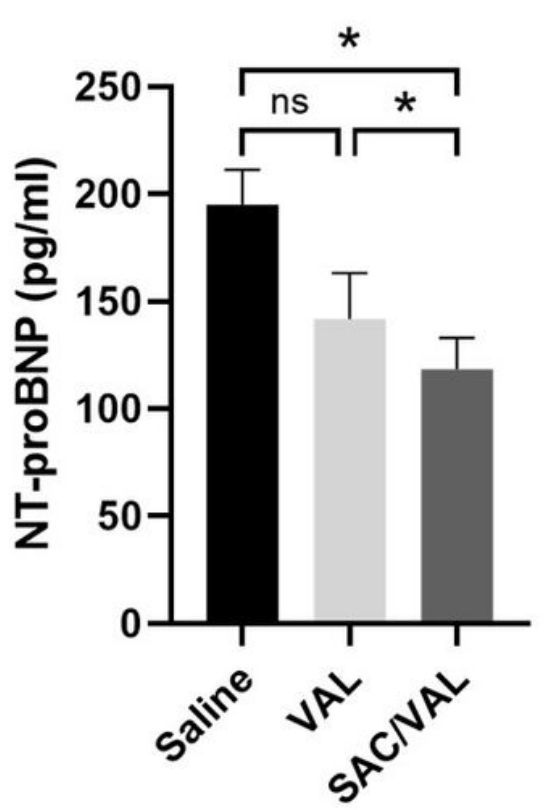

$\mathrm{F}$

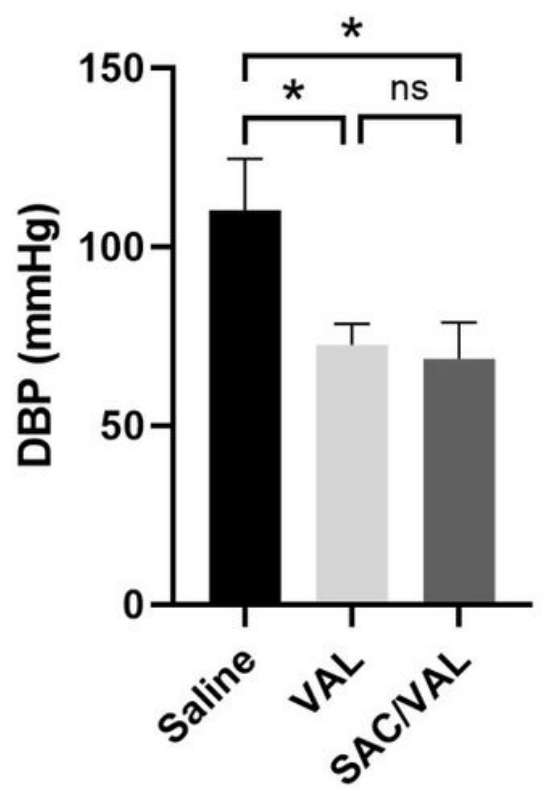

Figure 6

Sacubitril-valsartan decreases AF susceptibility and duration in Ang-II-infused rats. (A) Representative recordings of rat endocardial electrogram during burst pacing (upper panel), atrial fibrillation (middle panel) and normal sinus rhythm (lower panel). (B and C) Quantitative analysis AF inducibility and duration migration for these three groups. Data are expressed as the mean $\pm S D$ ( $n=6$ rats per group). *P $<0.05$ vs another group. AF: Atrial fibrillation. VAL: Valsartan; SAC/VAL: Sacubitril-valsartan. 


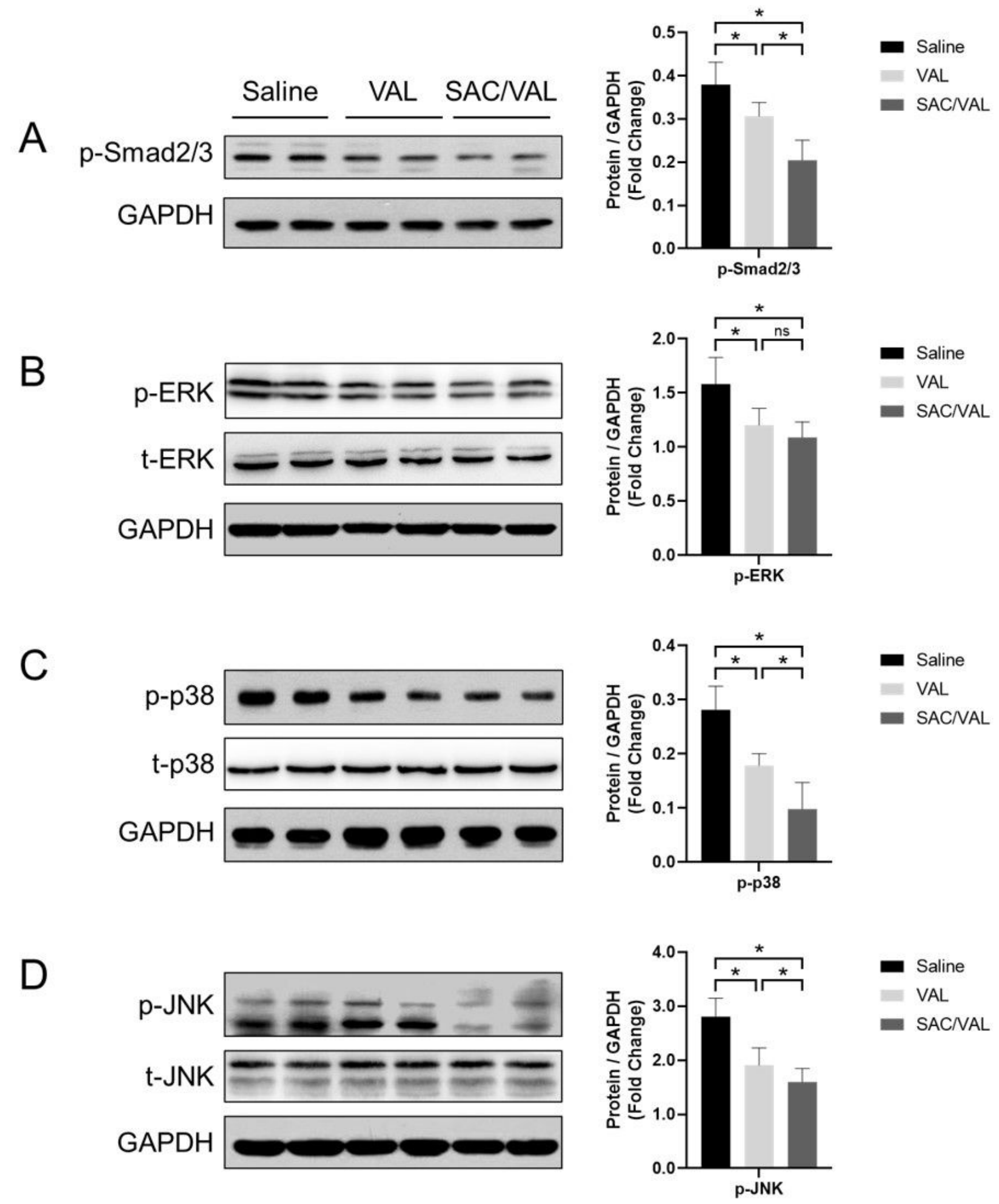

Figure 7

Sacubitril-valsartan inhibits the Ang-II-induced phosphorylation of Smad2/3, ERK1/2, p38 and JNK in rats. (A, B, C and D) Proteins extracted from atriums were detected by Western blot analysis (right) and quantification (left) using anti-phospho-Smad2/3, anti-phospho-ERK1/2, anti-ERK1/2, anti-phospho-p38, anti-p38, anti-phospho-JNK, and anti-JNK. GAPDH was used as loading control. Data are expressed as the mean $\pm S D$ ( $n=6$ samples per group). ${ }^{*} P<0.05$ vs another group; $n s$, not significant. $p-S m a d 2 / 3$, 
phosphorylated small mother against decapentaplegic 2/3; p-ERK1/2, phosphorylated extracellular signal-regulated kinase 1/2; t-ERK1/2, total extracellular signal-regulated kinase 1/2; p-JNK, c-Jun Nterminal kinase; t-JNK, total c-Jun N-terminal kinase; GAPDH, Glyceraldehyde 3-phosphate dehydrogenase. 